\title{
INFLUENCE OF DIFFERENT VEHICLES ON THE ANTIBACTERIAL/ ANTIMICROBIAL EFFECTS OF CALCIUM HYDROXIDE
}

\author{
M.R. Ganesh ${ }^{1}$, Shubhra Baranwal ${ }^{2}$, Nishtha Chauhan ${ }^{3}$
}

\begin{abstract}
HOW TO CITE THIS ARTICLE:
M.R. Ganesh, Shubhra Baranwal, Nishtha Chauhan. "Influence of different vehicles on the antibacterial/ antimicrobial effects of calcium hydroxide". Journal of Evolution of Medical and Dental Sciences 2013; Vol2, Issue 40, October 07; Page: 7598-7600.
\end{abstract}

\begin{abstract}
This study was done to find out the influence of three different vehicles on the antimicrobial activity of calcium hydroxide against the four microorganisms commonly seen in the infected pulp of the root canals. For this study cell culture plates with solid media were used. The results of the study showed that all calcium hydroxide pastes were effective in killing the microorganisms tested but at different times. Amongst them only calcium hydroxide CMCP pastes and glycerin pastes were the most effective against the microorganisms tested.
\end{abstract}

KEY WORDS: calcium hydroxide, camphorated mono chlorophenol, glycerine.

INTRODUCTION: Successful endodontic therapy depends on three basic phases laid down in the field of endodontics namely diagnostic phase, preparatory phase and obturative phase. Disinfection of the root canal is obtained by the combined effect of biomechanical preparation, irrigation and intra canal medicament [6,7]. Some studies have reported that microorganisms may remain viable even after complete chemo-mechanical preparation and thus affects the result of root canal treatment. The use of intra canal medicament is commonly advised in the treatment of non vital teeth to control the growth of microorganisms in between visits and to prevent reinfection [2,3]. Calcium hydroxide has been widely used as intra canal medicament because of its anti microbial action, ionic dissociation, high $\mathrm{pH}$ and enzyme inhibiting activity $\left[{ }^{8,9}, 10\right]$.Studies have observed that calcium hydroxide is superior to CMCP in effectiveness against microorganisms and is not an irritant to the pulp and periapical tissues even if it remains in paste form for longer time. Sigueria showed that calcium hydroxide paste with saline solution was ineffective in eliminating E. faecalis even after 1 week of exposure; on the other hand calcium hydroxide/CMCP/glycerin paste effectively killed microorganisms within 1 hour of exposure except for E. faecalis that required 24 hour of exposure. Calcium hydroxide mixed with distilled water, saline or glycerine has little or no antimicrobial effects [1]. Calcium hydroxide mixed with CMCP demonstrated pronounced antibacterial activity. Hence the aim of this study was to find out the influence of different vehicles on the antimicrobial effects of calcium hydroxide.

MATERIALS AND METHOD: Considering the microflora of infected root canal four bacterial strains were obtained namely Staph aureus, pseudomonas aeruginosa, enterococcus faecalis, and streptococcus sanguis. Calcium hydroxide powder was mixed with different vehicles such as normal saline, glycerin and CMCP-Glycerin. The ratio used in this study to prepare paste was 1:1 that is 1 gm calcium hydroxide powder with 1cc of appropriate liquid. Such prepared pastes were divided into different groups namely control $(0.85 \%$ saline $)$ group, calcium hydroxide- saline solution, calcium hydroxide-CMCP-glycerin and calcium hydroxide-glycerin. Four walls of $5 \mathrm{~mm}$ diameter 
were cut on each solid media and a standard loop with an internal diameter of $4 \mathrm{~mm}$, which could deliver $0.01 \mathrm{ml}$ of the suspension of culture of test organisms, then spread across the culture plate. The walls were then filled with calcium hydroxide pastes and culture plates were then incubated at $37^{\circ} \mathrm{C}$.

Growth and multiplication of microorganisms at the time intervals of 10 minutes, 1 hour, 1 day and 3 days were measured and the results observed.

RESULT: All the pastes were effective in killing the microorganisms tested but at different time. The calcium hydroxide/CMCP/Glycerin paste was very effective against the microorganisms tested.

DISSCUSSION: Calcium hydroxide is effective in eliminating the microorganisms from the infected root canals. It has bacteriocidal effect and has the capacity to neutralize the bacterial endotoxins. The antibacterial effectiveness of calcium hydroxide is related to its ionic dissociation creating alkaline environment [3]. However, time required to kill the microorganisms was different/similar when compared with other studies [4,5]. Calcium hydroxide is bacteriostatic at lower concentration and bacteriocidal at higher concentration. Hydroxyl ion concentration is higher when the paste is applied and it's lower when diffuse to tissues. The present study shows that all the different calcium hydroxide pastes were effective in killing the microorganisms but required different time span. Amongst all calcium hydroxide/CMCP/ Glycerin paste rapidly killed the microorganisms. This indicates that CMCP enhanced the antibacterial effectiveness of calcium hydroxide paste. CMCP cannot be considered as a vehicle to calcium hydroxide due to its tissue irritating property, but only as an additional medication [7]. This study demonstrated that calcium hydroxide/CMCP/ Glycerin paste is found to be most effective anti-bacterial intra-canal medicament tested and its efficacy in vivo requires further investigation.

\section{REFERENCES:}

1. Difore PM. Peters DD, Jean AS, Lewis M. The antibacterial effect of calcium hydroxide apexification pastes on streptococcus sanguis. Oral surgery 1982; 54:560-565.

2. Estrela C. In vitro determination of direct antimicrobial effect of calcium hydroxide. JOE1998; 24.

3. Estrela C. Mechanism of action of calcium and hydroxyl ions of calcium hydroxide on tissue and bacteria. Brazil dental J.1995; 6; 85-89.

4. Fabricus et al. Predominant indigenous oral bacteria isolated from infected root canal after varied time of closure. Scand J Dent R, 1982; 90-134.

5. Frederick R. Antibacterial efficacy of calcium hydroxide, iodine, potassium iodide, betadine scrub with and without surfactant against E. faecalis in vitro. Oral Surg Oral Med Oral Pathol Oral radio, Endodo 2004; 98.

6. Gomes in vitro anti microbial activity of several concentrations of sodium hypochlorite and chlorhexidine gluconate in the elimination of E. faecalis. JOE 2003; 34.

7. Harrison IW and Madonia JV. Antimicrobial efficacy of para chlorophenol. Oral Surg 1970; 30; 267-275.

8. Rivera EM, William K. Placement of calcium hydroxide in stimulated canals, comparison of glycerin versus water. J Endod 1994; 20; 445-448. 
9. Sylvia TS, Francis Research, Bhat KS. Effect of vehicles on the $\mathrm{pH}$ of calcium hydroxide and the release of Calcium ions, Oral Surgery 1995; 80; 459-464.

10. Sundquist G. Ecology of root canal flora. J Endod 1992; 18; 427.

\section{AUTHORS:}

1. M.R. Ganesh

2. Shubhra Baranwal

3. Nishtha Chauhan

\section{PARTICULARS OF CONTRIBUTORS:}

1. Reader, Department of Endodontics, Institute of Dental Sciences, Bareilly, Uttar Pradesh.

2. $2^{\text {nd }}$ Year Student, Department of Conservative and Endodontics, Institute of Dental Sciences, Bareilly, Uttar Pradesh.

3. Final Year Student, Department of Conservative and Endodontics, Institute of Dental Sciences, Bareilly, Uttar Pradesh.

\section{NAME ADDRESS EMAIL ID OF THE} CORRESPONDING AUTHOR:

Dr. M.R. Ganesh, CST, Back of G.P.O, Mumbai - 01.

Email- watvrukshpippa1909@gmail.com

Date of Submission: 21/09/2013.

Date of Peer Review: 22/09/2013.

Date of Acceptance: 26/09/2013.

Date of Publishing: 01/10/2013. 